\title{
Positron emission mammography with tomographic acquisition using dual planar detectors: initial evaluations
}

\author{
Mark F Smith ${ }^{1}$, Raymond R Raylman ${ }^{2}$, Stan Majewski ${ }^{1}$ \\ and Andrew G Weisenberger ${ }^{1}$ \\ ${ }^{1}$ Thomas Jefferson National Accelerator Facility, Newport News, VA 23606, USA \\ ${ }^{2}$ Center for Advanced Imaging, Department of Radiology, West Virginia University, \\ Morgantown, WV 26506, USA \\ E-mail: mfsmith@jlab.org
}

Received 27 October 2003

Published 19 May 2004

Online at stacks.iop.org/PMB/49/2437

DOI: $10.1088 / 0031-9155 / 49 / 11 / 022$

\begin{abstract}
Positron emission mammography (PEM) with tomographic acquisition using dual planar detectors rotating about the breast can obtain complete angular sampling and has the potential to improve activity estimation compared with PEM using stationary detectors. PEM tomography (PEMT) was compared with stationary PEM for point source and compressed breast phantom studies performed with a compact dual detector system. The acquisition geometries were appropriate for the target application of PEM guidance of stereotactic core biopsy. Images were reconstructed with a three-dimensional iterative maximum likelihood expectation maximization algorithm. PEMT eliminated blurring normal to the detectors seen with stationary PEM. Depth of interaction effects distorted the shape of the point spread functions for PEMT as the angular range from normal incidence of lines of response used in image reconstruction increased. Streak artefacts in PEMT for large detector rotation increments led to the development of an expression for the maximum rotation increment that maintains complete angular sampling. Studies with a compressed breast phantom were used to investigate contrast and signal-to-noise ratio (SNR) trade-offs for different sized spherical tumour models. PEMT and PEM both had advantages depending on lesion size and detector separation. The most appropriate acquisition method for specific detection or quantitation tasks requires additional investigation.
\end{abstract}




\section{Introduction}

Positron emission mammography (PEM) is a method for imaging the uptake of positron-emitting radiotracers (e.g. F-18 fluorodeoxyglucose) in the breast and is being investigated as a means for detecting breast cancer (Weinberg et al 1996, Murthy et al 2000, Levine et al 2003). PEM is typically accomplished with two parallel planar detectors positioned above and below a compressed breast (Thompson et al 1994, Weinberg et al 1996, Doshi et al 2000, Raylman et al 2000), though other configurations are also being investigated (Freifelder and Karp 1997, Virador et al 1998). Clinical acquisitions have been performed with the dual planar detector configuration (Weinberg et al 1996, Murthy et al 2000, Turkington et al 2002) and with translation of rectangular detectors to achieve a larger field of view than is possible with detectors in a fixed position (Weinberg et al 2002). A PEM system has been integrated with a stereotactic x-ray mammography system to obtain combined functional and anatomical imaging of breast cancers (Adler et al 2003).

Image reconstruction for dual detector PEM can be accomplished by simple backprojection (laminography) (Thompson et al 1995) or iterative methods (Moses and Qi 2003, Smith et al 2003). Image quality is improved with iterative reconstruction due to its superior method of modelling data acquisition and solving the limited angle tomography problem. With either reconstruction method the limited angular range of the lines of response (LORs) for dual detector PEM causes blurring normal to the detector, which hinders accurate three-dimensional (3D) activity estimation. We have been investigating the application of PEM to guide stereotactic core biopsy (Raylman et al 2001a). In an effort to improve spatial resolution normal to the detectors, PEM acquisition with multiple detector positions over a $+15^{\circ}$ to $-15^{\circ}$ angular range was recently investigated in a phantom experiment and was found to improve signal-to-noise versus resolution trade-offs for small $(<1 \mathrm{~cm}$ diameter $)$ lesions (Smith et al 2002).

Positron emission mammography with tomographic acquisition (positron emission mammotomography or PEMT) and complete angular sampling has the potential to improve breast imaging over PEM with stationary dual planar detectors by reducing blurring normal to the detectors. The goal of this paper is to make an initial experimental evaluation of $3 \mathrm{D}$ breast imaging with tomographic acquisitions using dual planar detectors. It has previously been proposed to achieve complete angular sampling for positron breast imaging by completely surrounding the breast by a rectangular detector (Virador et al 1998, Huesman et al 2000), though construction of this instrument is still underway. A simulation study comparing this rectangular detector with a dual planar detector system using the Fisher information matrix has shown that the rectangular system with depth of interaction capability has a higher signal-to-noise ratio for detection tasks and a lower bias at a given noise level for quantitation tasks (Qi et al 2002). For these simulations the cross section of the rectangular detector was $9.6 \mathrm{~cm} \times 7.2 \mathrm{~cm}$ and the dual planar detectors were $16.8 \mathrm{~cm} \times 7.2 \mathrm{~cm}$ with a separation of $7.2 \mathrm{~cm}$.

In this current contribution PEMT and PEM coincidence data were acquired using a compact dual detector system built with pixellated scintillator arrays (Raylman et al 2000). Studies with point sources were used to quantify the width of the point spread function (PSF) for stationary PEM and PEMT. Studies with a compressed breast phantom were used to investigate contrast and signal-to-noise ratio (SNR) trade-offs for different sized spherical tumour models. A geometric analysis led to the development of an equation for the maximum detector rotation increment that can be made that still maintains complete angular sampling for tomographic acquisitions. 
(a)

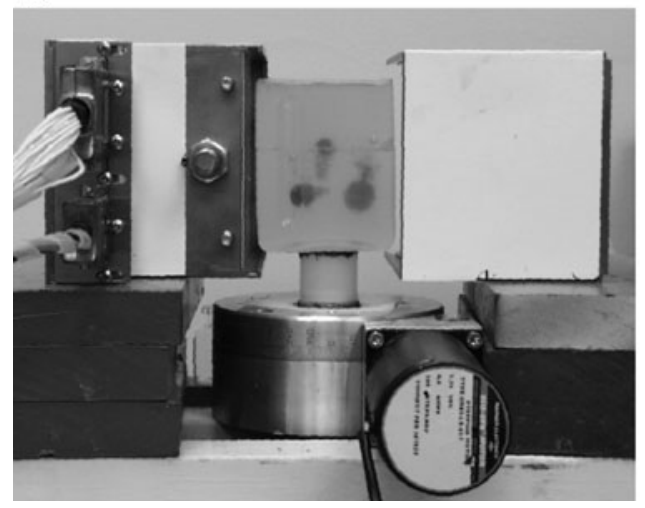

(b)

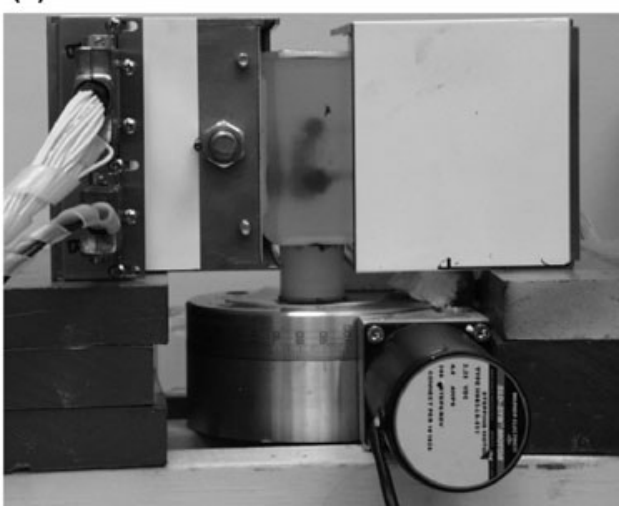

Figure 1. PEM detectors, rotation stage and compressed breast phantom used for the PEM and PEMT phantom studies. (a) Side view of the detectors with a separation of $10 \mathrm{~cm}$ for tomographic acquisitions and the compressed breast phantom at a $90^{\circ}$ rotation angle. (b) Compressed breast phantom at a zero degree rotation angle with a detector separation of $6.3 \mathrm{~cm}$ for one of the static PEM acquisitions.

\section{Methods}

\subsection{Data acquisition}

List-mode coincidence data were acquired with a dual detector PEM system having gadolinium oxyorthosilicate (GSO) crystal elements (figure 1) (Raylman et al 2000). The field of view of each detector head was $10 \times 10 \mathrm{~cm}^{2}$. The individual crystals were $3.1 \times 3.1 \times 10 \mathrm{~mm}^{3}$ with a pitch of $3.3 \mathrm{~mm}$. A $1 \mathrm{~cm}$ thick acrylic light guide coupled the scintillation array to a $4 \times 4$ array of Hamamatsu R7600-C8 position-sensitive photomultiplier tubes. The analogue outputs of the two detector heads were read out by a CAMAC-based data acquisition system utilizing two LeCroy FERA analogue to digital converters (ADCs) (LeCroy Corporation, Chestnut Ridge, NY, USA). The control program and system interface were written using Kmax (Sparrow Inc, Daytona Beach, Florida, USA) running on an Apple Macintosh G4 computer. This detector system has a maximum coincidence rate of about 2450 events/s with real-time display of backprojection reconstructed images; this event rate is limited by the speed of the ADCs and the control computer.

Point source acquisitions were made with a cross arrangement of $\sim 1.5 \mathrm{~mm}$ diameter ceramic beads that had been soaked in an F-18 solution. Nine beads were spaced at a $2 \mathrm{~cm}$ interval along each of two perpendicular arms. The cross was placed on a rotation stage, oriented perpendicular to the detector heads and centred in the field of view. The distance between the front scintillator surfaces was $11 \mathrm{~cm}$. Coincidence data were acquired at 18 positions of the rotation stage with a rotational increment of $20^{\circ}$. At each rotation stage position coincidence data were acquired for $60 \mathrm{~s}$. The purpose of performing a $360^{\circ}$ rotation rather than only a $180^{\circ}$ rotation was to average residual inhomogeneities in detector response. Note that if a centre of rotation correction is required then in general the lines of response (LORs) through an object will not be the same at angles $\theta$ and $\theta+180^{\circ}$. Two tomographic datasets were created, the first with coincidence data from a $60^{\circ}$ rotation increment (rotation stage positions $0^{\circ}, 60^{\circ}, 120^{\circ}, 180^{\circ}, 240^{\circ}$ and $300^{\circ} ; 6$ min total time) and the second with coincidence data from a $20^{\circ}$ rotation stage increment $\left(0^{\circ}, 20^{\circ}, \ldots, 340^{\circ} ; 18 \mathrm{~min}\right.$ total time). 
A compressed breast phantom was made to model the clinical setting for PEM-guided sterotactic core biopsy (figure 1). A plastic mould $7.0 \mathrm{~cm}$ wide, $8.8 \mathrm{~cm}$ high and $3.4-4.6 \mathrm{~cm}$ thick (there was a slight curvature) was filled with a gelatin mixture containing an F-18 concentration of $0.055 \mu \mathrm{Ci} \mathrm{ml}^{-1}\left(0.20 \mathrm{kBq} \mathrm{ml}^{-1}\right)$ as background activity. Breast tumours were modelled by 9,12 and $15 \mathrm{~mm}$ diameter fluid-filled spheres with radioactivity concentration $0.55 \mu \mathrm{Ci} \mathrm{ml} l^{-1}\left(2.04 \mathrm{kBq} \mathrm{ml}^{-1}\right)$. The centres of the spheres were positioned in a plane that was approximately parallel to the detector surface for PEM acquisitions (angle $0^{\circ}$ for PEMT acquisitions). The distances between the front scintillator surfaces were $6.3,8.0$ and $10.0 \mathrm{~cm}$ for the static PEM acquisitions and $10.0 \mathrm{~cm}$ for the tomographic acquisitions. The aluminium front plate of each detector was almost touching the phantom with the $6.3 \mathrm{~cm}$ detector separation; this separation is greater than $4.6 \mathrm{~cm}$ because the plastic walls of the phantom were about $1 \mathrm{~mm}$ thick and the front surfaces of the scintillator arrays were about $7 \mathrm{~mm}$ behind the aluminium face plate. The detector separation for tomography modelled the minimum possible with a LORAD stereotactic breast biopsy system (Hologic Inc., Bedford, MA, USA), which has a $10 \mathrm{~cm}$ wide compression paddle.

Coincidence data for the breast phantom studies were acquired to simulate $12 \mathrm{~min}$ acquisitions for PEM and PEMT as follows. The phantom contained sufficient activity that the maximum coincidence count rate was achieved at all detector separations, thus all time-equivalent datasets contained approximately the same number of raw coincidence events before application of energy window thresholds and any acceptance angle restrictions.

The PEMT studies were acquired using the step-and-shoot method with rotation stage increments of $20^{\circ}$ from $0^{\circ}$ to $340^{\circ}$. Data were acquired for $120 \mathrm{~s}$ at each rotation position. These coincidence data were used to create two time-equivalent datasets modelling $12 \mathrm{~min}$ tomographic studies. Set 1 used coincidence data from a $60^{\circ}$ rotation increment (rotation stage positions $0^{\circ}, 60^{\circ}, 120^{\circ}, 180^{\circ}, 240^{\circ}$ and $300^{\circ} ; 120 \mathrm{~s}$ of data from each position), while set 2 used coincidence data from a $20^{\circ}$ rotation increment (rotation stage positions $0^{\circ}, 20^{\circ}, \ldots$, $340^{\circ} ; 40 \mathrm{~s}$ of data from each position). For some of the PEM studies with a fixed detector position, the activity had decayed so that the coincidence rate was below the detector rate maximum. In these cases data were acquired for a sufficiently long time to allow creation of datasets at all detector separations modelling $12 \mathrm{~min}$ acquisitions at the maximum coincidence rate.

\subsection{Image reconstruction}

Events along each line of response (LOR) were tallied from the list-mode data. An iterative maximum likelihood expectation maximization (MLEM) algorithm (Shepp and Vardi 1982, Lange and Carson 1984) was employed for three-dimensional image reconstruction using data from all LORs simultaneously, with update formula

$$
s_{j}^{n+1}=s_{j}^{n}\left(1 / \sum_{m, i} c_{m i j}\right)\left(\sum_{m, i} \frac{c_{m i j} L_{m i}}{\sum_{j} c_{m i j} s_{j}^{n}}\right)
$$

(Huesman et al 2000, Qi and Huesman 2002, Smith et al 2003). In this equation $s_{j}^{n}$ is the amplitude of source voxel $j$ at iteration $n ; c_{m i j}$ is the system matrix element characterizing detection of a true coincidence event for LOR $i$ at detector angle $m$ from activity in source voxel $j$; and $L_{m i}$ is the number of true coincidence events detected for LOR $i$ at detector angle $m$. The starting source distribution was uniform and 20 iterations were performed.

The system matrix modelled attenuation for the breast phantom but not for the point source studies. No compensation was made for randoms or scatter. Mid-crystal positions 
were used for LOR ray-tracing calculations. The energy window was $400-700 \mathrm{keV}$ and the reconstructed voxel size was $\left(1.65 \mathrm{~mm}^{3}\right)$. The support region for image reconstruction was a cube 60 voxels $(9.9 \mathrm{~cm})$ on a side for the point source studies and a $10 \mathrm{~cm} \times 10 \mathrm{~cm} \times 5 \mathrm{~cm}$ region for the compressed breast phantom. Preliminary image reconstructions of the breast phantom showed artefacts possibly related to depth of interaction effects, so the incidence angle of coincidence events used in image reconstruction of these phantoms was limited to $40^{\circ}$ from normal incidence. Post-reconstruction three-dimensional Gaussian smoothing filters (2.0-4.0 mm FWHM) were applied to the reconstructed images of the breast phantoms after all iterations were completed for regularization and noise suppression (Nuyts and Fessler 2003).

Image reconstruction is a case of limited angle tomography for PEM with a stationary detector. For the tomographic acquisitions angular sampling is complete if the angular rotation increment is sufficiently small (see section 3.3). Tomographic image reconstruction is similar to the PET case for two large planar rotating detectors operating in coincidence mode (Muehllehner et al 1976, Patton and Turkington 1999, Levkovitz et al 2001).

\subsection{Data analysis}

Resolution of the point sources was measured in orthogonal directions in transaxial reconstructed image slices. Gaussian curves were fit to profiles and the full width at half maximum (FWHM) was computed. For the reconstructed breast phantom images, the contrast $(C)$ and signal-to-noise ratios (SNRs) were computed using the expressions $C=(T-B) / B$ and SNR $=(T-B) / \sigma(B)$, where $T$ was the mean activity in a spherical tumour model region of interest (ROI), $B$ was the mean activity in a background region and $\sigma(B)$ was the standard deviation of voxel values in the background region. The planes used for ROI analysis were slices parallel to the detector plane in the PEM orientation (parallel to the detector plane at $0^{\circ}$ for PEMT). The ROIs for the spheres were circles containing 9, 13 and 21 voxels for the 9, 12 and $15 \mathrm{~mm}$ spheres, respectively. Rectangular regions adjacent to each sphere were used for the background regions. Four rectangles were used for the $9 \mathrm{~mm}$ sphere while only three rectangles were used for the 12 and $15 \mathrm{~mm}$ spheres since they were close to the side of the phantom. Each rectangular background region averaged 56 voxels. The ROI analysis used voxels from three adjacent image slices, with the centre slice chosen individually for each sphere.

\subsection{Analysis of angular sampling}

Streak artefacts were observed in image reconstructions for the $60^{\circ}$ increment dataset, which led to an analysis of conditions for complete angular sampling in tomographic PEM acquisitions. Let two planar detectors be oriented parallel to each other with separation $D$ and detector width $L$ as depicted in the transaxial view of figure 2(a). Furthermore, let the axis of rotation (AOR) be at the centre of the field of view and let it be the origin of an $x-y$ coordinate system. The coordinates of a point $P$ in the field of view of the detectors can be given by $\left(x_{p}, y_{p}\right)=(R \cos \phi, R \sin \phi)$ where $R$ is the radial distance from the AOR and $\phi$ is the polar angle measured counterclockwise from the $x$-axis.

The greatest angle from normal incidence in the clockwise direction for a detectable coincidence event (figure 2(b)) is given by $\psi_{13, \min }=\min \left(\psi_{1}, \psi_{3}\right)$ and the greatest angle from normal incidence in the counterclockwise direction for a detectable coincidence event is given by $\psi_{24, \min }=\min \left(\psi_{2}, \psi_{4}\right)$. The potential extremal LORs can be obtained by tracing rays from the endpoints of the detectors indicated by points $P_{1}-P_{4}$ in figure 2(b) through point $P$. The 
(a)

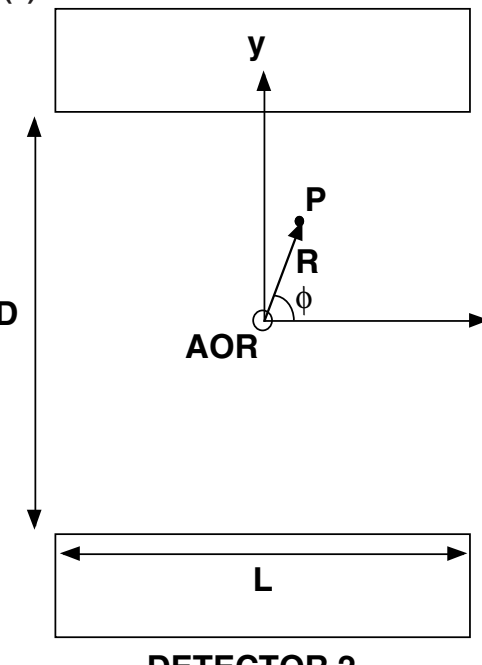

(b) DETECTOR 1

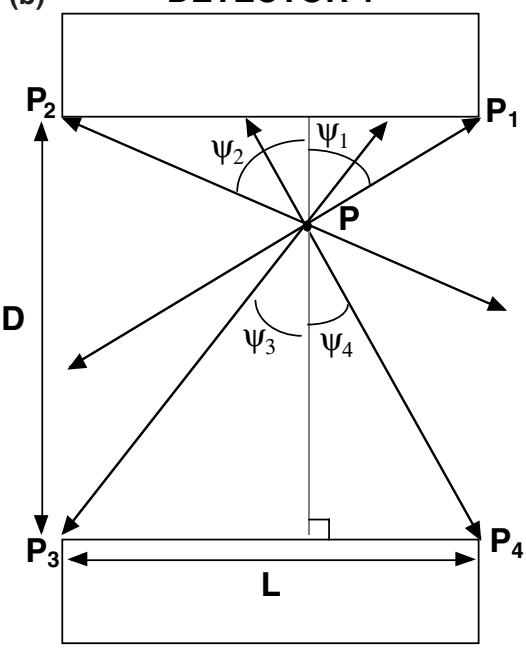

DETECTOR 2

Figure 2. Diagram of a two-detector PEM system perpendicular to the axis of rotation (AOR). (a) Location of point $P$ with respect to Euclidean and polar coordinate systems with origin at the AOR and (b) point $P$ with potential extremal lines of response and corresponding angles $\psi$ from normal incidence.

tangents of angles $\psi_{1}-\psi_{4}$ are given by

$$
\begin{aligned}
\tan \psi_{1} & =\frac{L / 2-R \cos \phi}{D / 2-R \sin \phi} \\
\tan \psi_{2} & =\frac{L / 2+R \cos \phi}{D / 2-R \sin \phi} \\
\tan \psi_{3} & =\frac{L / 2+R \cos \phi}{D / 2+R \sin \phi} \\
\tan \psi_{4} & =\frac{L / 2-R \cos \phi}{D / 2+R \sin \phi} .
\end{aligned}
$$

For a point $P$ at radius $R$ from the AOR we wish to know the smallest values reached by $\psi_{13 \text {,min }}$ or $\psi_{24 \text {,min }}$ at any polar angle $\phi$, for then the detector can be rotated by twice this angle and complete angular sampling can be obtained, i.e. no angles will be missed. Mathematically we wish to find, for a fixed $R, \psi_{\min }=\min _{\phi}\left(\min \left(\psi_{1}, \psi_{3}\right), \min \left(\psi_{2}, \psi_{4}\right)\right)$. Then the maximum rotation angle that still maintains complete sampling is $\theta_{\max }=2 \psi_{\min }$.

Without loss of generality it suffices, due to symmetry considerations, to find $\psi_{\min }$ in quadrant 1 where $0 \leqslant \phi \leqslant \pi / 2$. An examination of equations $(2 a)-(2 d)$ and figure 2 (b) shows that $\psi_{4}=\min \left(\psi_{1}, \psi_{2}, \psi_{3}, \psi_{4}\right)$. Since $0 \leqslant \psi_{4} \leqslant \pi / 2$ the minimum value of $\psi_{4}$ is found where $\tan \psi_{4}$ is a minimum. We find that

$$
\frac{\mathrm{d} \tan \psi_{4}}{\mathrm{~d} \phi}=\frac{R \sin \phi(R \sin \phi+D / 2)-R \cos \phi(L / 2-R \cos \phi)}{(R \sin \phi+D / 2)^{2}} .
$$


Some algebraic manipulation shows that this first derivative is zero where

$$
\begin{aligned}
& \sin \phi=\frac{L\left[L^{2}+D^{2}-4 R^{2}\right]^{1 / 2}-2 R D}{L^{2}+D^{2}} \\
& \cos \phi=\frac{D\left[L^{2}+D^{2}-4 R^{2}\right]^{1 / 2}+2 R L}{L^{2}+D^{2}} .
\end{aligned}
$$

The derivation assumes that $R<D / 2$ (the point is always between the detectors) and that $R<L / 2$ (the point is always in the field of view). The second derivative $\frac{d^{2} \tan \psi_{4}}{d \phi^{2}}$ is positive at this extremal point, indicating a local minimum. Combining (4a) and (4b) and the equation for $\psi_{\min }$ we find that angular sampling for point $P$ is complete if the detector rotates through $180^{\circ}$ and the detector rotation increment $\theta_{\text {rot }} \leqslant \theta_{\max }$ where

$$
\theta_{\max }=2 \arctan \left(\frac{L\left[L^{2}+D^{2}-4 R^{2}\right]^{1 / 2}-2 R D}{D\left[L^{2}+D^{2}-4 R^{2}\right]^{1 / 2}+2 R L}\right) .
$$

Angular sampling for the source region thus will be complete when all source activity is within a distance $R$ from the AOR and this condition is satisfied.

If image reconstruction is performed with an angular limit $\theta_{\text {limit }}$ on the acceptance angle from normal incidence of coincidence events, as might be done to avoid or minimize some depth of interaction effects, then complete angular sampling will be obtained only if $\theta_{\text {rot }} \leqslant \theta_{\max }$ and $\theta_{\text {rot }} \leqslant 2 \theta_{\text {limit }}$ are both satisfied.

\section{Results}

\subsection{Point source studies}

The point sources were resolved easily in all reconstructed images (figure 3(a), row 1). Radial and tangential resolution improved with iteration and remained about constant after iteration 6. For stationary (1 angle) PEM resolution averaged $0.89 \pm 0.18 \mathrm{~cm}$ FWHM normal to the detector and $0.37 \pm 0.07 \mathrm{~cm}$ parallel to it. For PEMT the resolutions were similar for the datasets with $60^{\circ}$ and $20^{\circ}$ rotation increments. Radial resolution was fairly constant and averaged $0.55 \pm 0.06 \mathrm{~cm}$ for the $60^{\circ}$ rotation increment data and $0.57 \pm 0.03 \mathrm{~cm}$ for $20^{\circ}$ increment data (figure 4(a)). Tangential resolution increased from $0.5 \mathrm{~cm}$ to $0.9 \mathrm{~cm}$ at $4 \mathrm{~cm}$ from the AOR with a mean $\sigma$ of $0.08 \mathrm{~cm}$ for the $60^{\circ}$ increment data and $0.03 \mathrm{~cm}$ for the $20^{\circ}$ increment data (figure 4(a)). Streak artefacts were visible in the $60^{\circ}$ rotation increment reconstructions for point sources $4 \mathrm{~cm}$ from the AOR.

The poorer tangential resolution was apparent in the cusp-like nature of the PSF for the point sources $4 \mathrm{~cm}$ from the AOR (figure 3(a), row 2). This may be due to a depth of interaction effect. As a test of this hypothesis, the point sources were reconstructed using coincidence events only from LORs with an incidence angle with the detector surface of less than $10^{\circ}$ from normal incidence. The images (figure 3(b)) and PSF widths (figure 4(b)) show that there was little difference on average between radial and tangential PSF widths for this case. These images were noisier than their counterparts in figure 3(a) due to fewer contributing coincidence events. Radial resolution averaged $0.48 \pm 0.06 \mathrm{~cm}$ for the $60^{\circ}$ rotation increment data and $0.48 \pm 0.08 \mathrm{~cm}$ for $20^{\circ}$ increment data. Tangential resolution averaged $0.54 \pm 0.18 \mathrm{~cm}$ for the $60^{\circ}$ increment data and $0.55 \pm 0.09 \mathrm{~cm}$ for the $20^{\circ}$ increment data.

Figure 5 provides some insight into the shape of the PSF near the edge of the tomographic field of view when all LORs are used in image reconstruction and why radial resolution is better than tangential resolution. When the detectors are at a position where a point source is 
(a)

ALL LORs
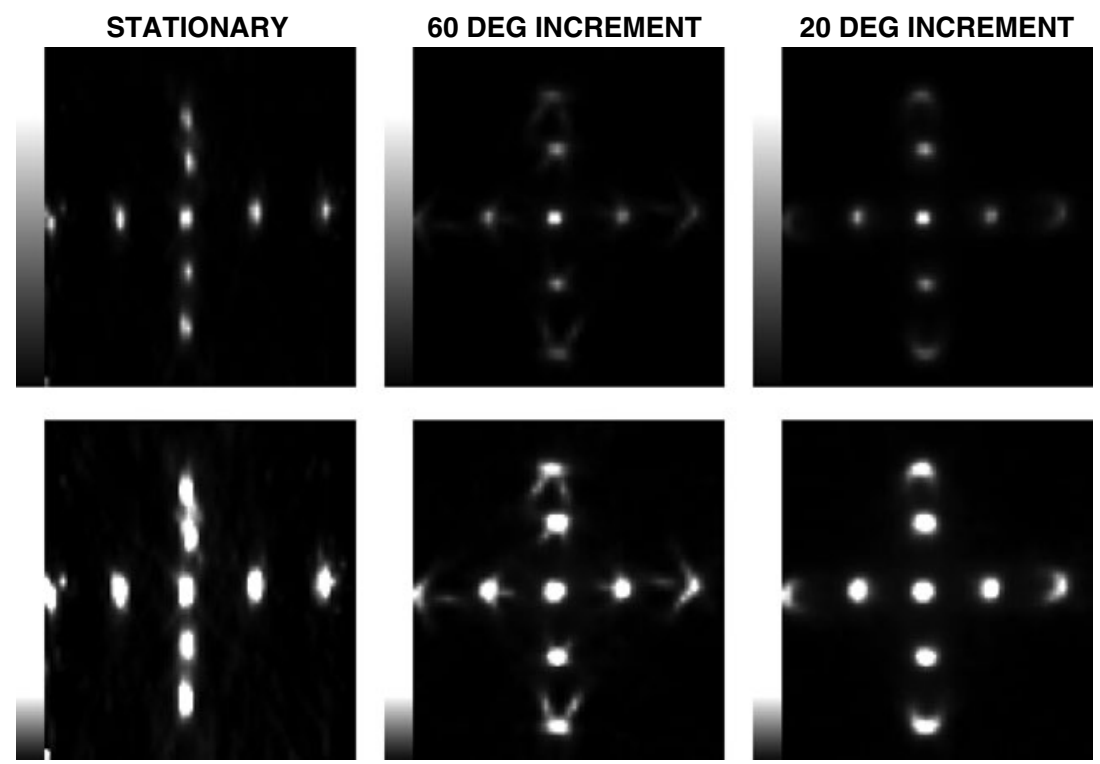

(b) 10 DEGREE MAXIMUM INCIDENCE ANGLE FROM NORMAL
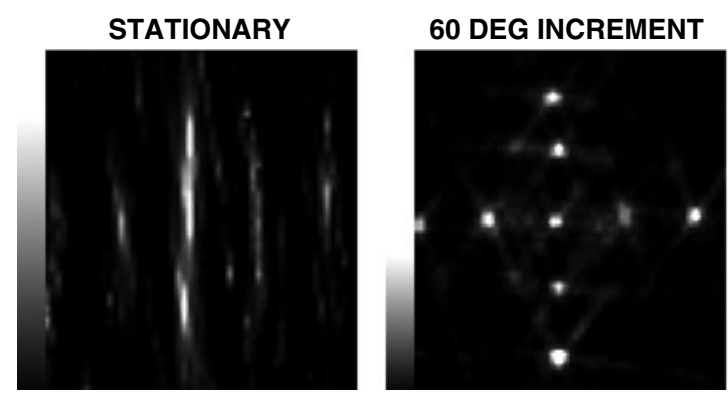

20 DEG INCREMENT

Figure 3. Reconstructed point sources for 1 angle (stationary detectors), $60^{\circ}$ and $20^{\circ}$ detector rotation increments (iteration 6). The slices are perpendicular to the axis of rotation. (a) All LORs are included in image reconstructions, row 1: grey scale at $80 \%$ of peak, row 2: grey scale at $20 \%$ of peak. (b) Only LORs with an incidence angle of less than $10^{\circ}$ from normal incidence are included in image reconstructions. From left to right, grey scale is at $80 \%, 40 \%$ and $40 \%$ of peak. The image for stationary PEM is noisy due to a limited number of events for this small angular limit.

close to midway between the detectors, the LORs are close to normal incidence, intercrystal penetration effects are small, and backprojected rays pass close to the point of positron emission (figure 5(a)). Viewed as a contribution to the PSF, the resolution in the radial direction is well constrained. When the detectors have rotated so that the point source is close to one of the detector heads, extremal raypaths for annihilation photons pass through several crystals (figure 5(b)). These photons could undergo photoelectric interactions in one of several crystals. The dashed lines show bounding bands for the LORs that would be used in image reconstruction. These bands are broader than those for raypaths closer to normal incidence; thus tangential resolution is not as well constrained as radial resolution. Of course the PSF is due to the combined effect of annihilation raypaths at all angles and for all detector positions. If the incidence angle from normal is limited in image reconstruction to some small angle, 


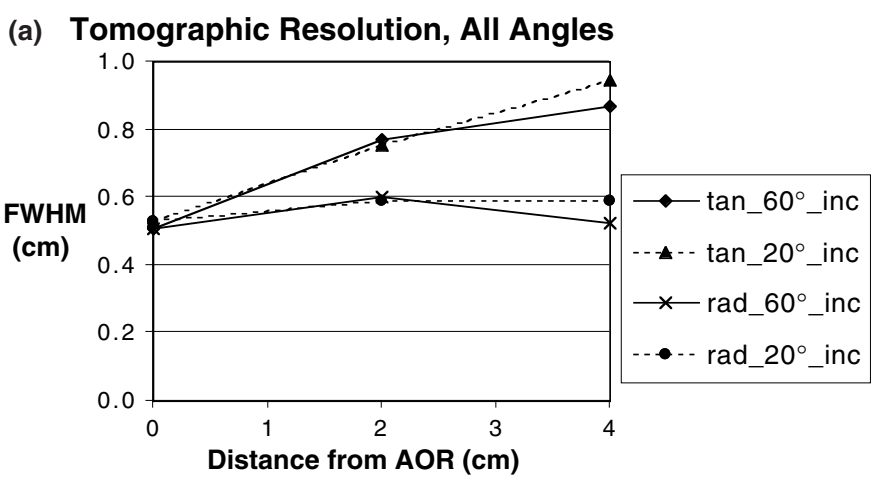

(b) Tomographic Resolution, $10^{\circ}$ Max Ang

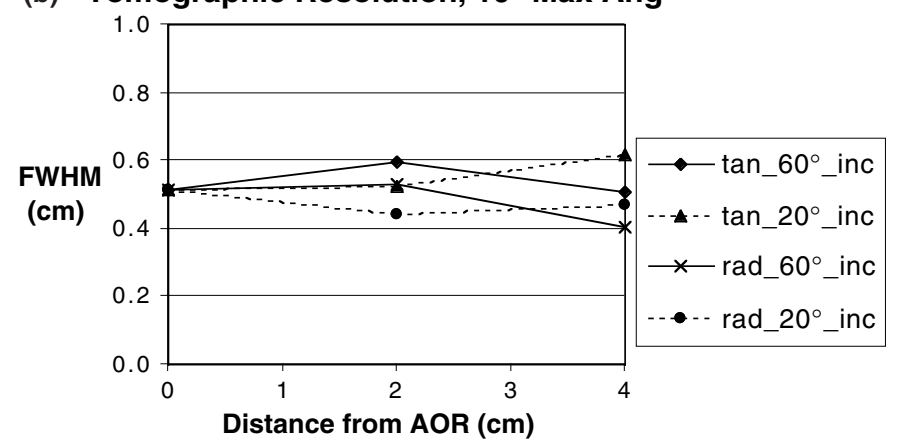

Figure 4. Radial and tangential tomographic resolution (FWHM) for the point source experiment (iteration 6) for (a) all LORs included in image reconstruction and (b) only LORs with a maximum angle from normal incidence of $10^{\circ}$. The rotation increment of the detector is indicated in the legend.

then the bounding bands will be about the same for positions in figures 5 (a) and (b) and so the PSF will be more isotropic. This depth of interaction effect could also be mitigated by using thinner crystals at the expense of sensitivity.

\subsection{Breast phantom}

Reconstructions of the compressed breast phantom showed excellent lesion visualization for all PEM and PEMT acquisitions (figure 6). There are streak artefacts and blurring between the detectors in transaxial slices for the single position PEM acquisitions but not for tomographic acquisitions, as expected (figures 6(b) and (c)). The tomographic images accurately show the cross section of the breast phantom, roughly a rectangular shape with slightly curved sides (two rightmost columns in figures 6(b) and (c)). For stationary PEM the image fills the $10 \times$ $10 \times 5 \mathrm{~cm}^{3}$ box-shaped support region for source activity used in image reconstruction. The number of coincidence events within the $40^{\circ}$ acceptance angle and the 400-700 keV energy window was 268,652 and 384, 498 and 378,720 for PEM with $6.3,8$ and $10 \mathrm{~cm}$ detector separations, respectively. There are about $30 \%$ fewer events for the $6.3 \mathrm{~cm}$ detector separation because the detector is rate-limited and a greater fraction of the detected events occur with larger incidence angles from normal incidence. The number of events was 354, 364 for PEMT with a $60^{\circ}$ rotation increment and 356,195 for PEMT with a $20^{\circ}$ angular increment. 
(a)

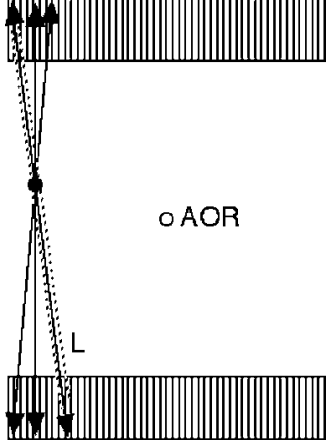

(b)

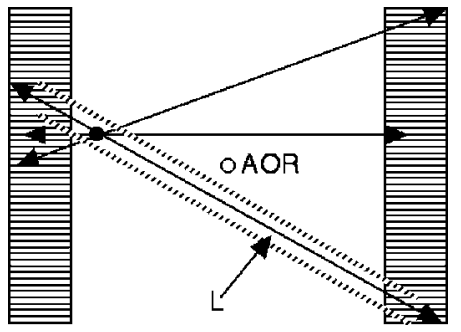

Figure 5. View through a plane normal to the axis of rotation of a dual detector PEM system showing the scintillation crystal array and a point source. When the point is near the edge of the field of view for PEM tomography, intercrystal penetration and depth of interaction effects for extremal LORs are less pronounced at detector positions where (a) the point is close to midway between the detectors compared with those where (b) the point is close to one of the detector heads. The dashed lines show bounding bands for the LORs that would be used in image reconstruction for annihilation photons with paths along line $L$ but undergoing photoelectric absorption in different detector crystals.

The SNR versus contrast curves (figure 7) show complex relationships between the performance of PEM and PEMT for different sphere sizes and for different PEM detector separations; however, some general conclusions can be drawn. For all acquisition methods, SNR decreases and contrast increases as the width of the Gaussian smoothing filter decreases, as expected. The PEMT curves for $20^{\circ}$ and $60^{\circ}$ rotation increments are close for all three spheres and the differences are likely due to Poisson noise in the coincidence data that propagates into the reconstructed images.

Consider first the curves for the 12 and $15 \mathrm{~mm}$ spheres. For PEM acquisition, better contrast and to some extent better SNR are obtained with smaller detector separations. This may be due to a greater angular range of LORs available for reconstruction over more of the volume of the phantom, enabling activity throughout the source region to be better estimated, even given the $40^{\circ}$ limit from normal incidence. For the $15 \mathrm{~cm}$ sphere, contrast for a given resolution is greater for $6.3 \mathrm{~cm}$ PEM than for PEMT; for the $12 \mathrm{~mm}$ sphere this is true for both 6.3 and $8 \mathrm{~cm}$ PEM. The reason for this difference is not immediately apparent. SNR is greater for PEMT than for PEM at poorer resolutions and similar at better resolutions. PEMT with a detector separation of $10 \mathrm{~cm}$ is always superior to PEM with a detector separation of $10 \mathrm{~cm}$. Contrast is higher at poorer resolutions for the $15 \mathrm{~mm}$ sphere than for the $12 \mathrm{~mm}$ sphere, as expected, though with the $2 \mathrm{~mm}$ FWHM Gaussian smoothing filter the contrast of the $12 \mathrm{~mm}$ sphere approached that of the $15 \mathrm{~mm}$ sphere, also as expected. For the $9 \mathrm{~mm}$ sphere all of the SNR versus contrast curves almost overlay each other except for that of $6.3 \mathrm{~cm}$ PEM, where SNR is smaller. This could be related to the fewer coincidence events in the energy and 
(a)

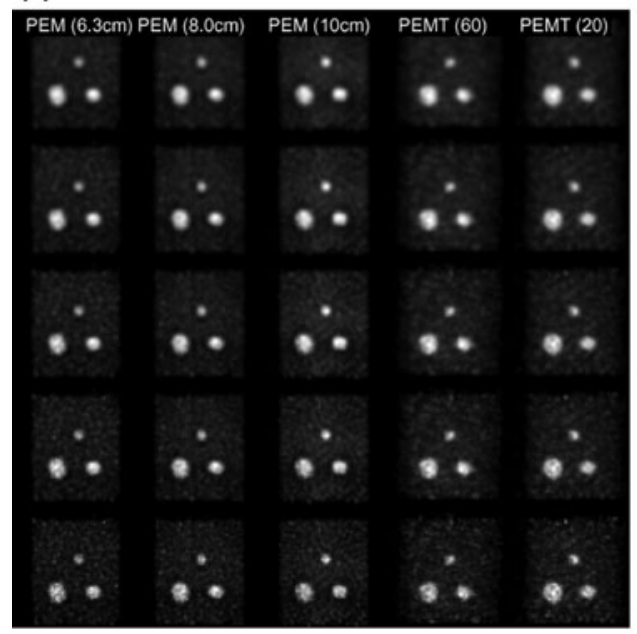

(b)

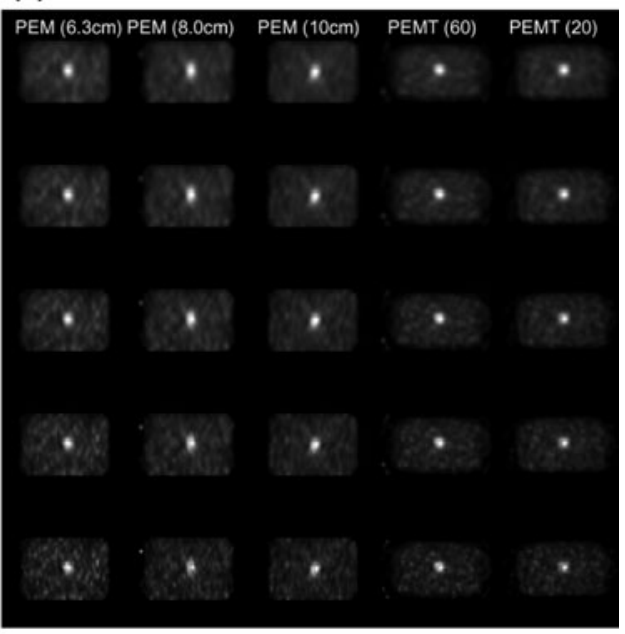

(c)

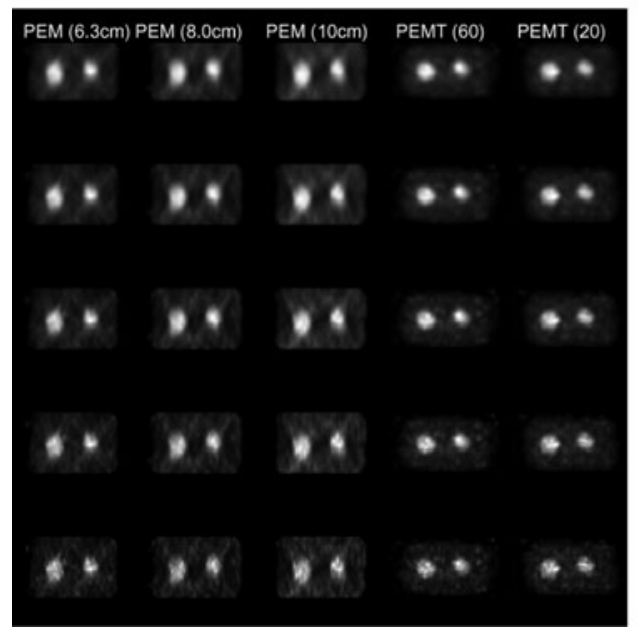

Figure 6. Slices through image reconstructions of the compressed breast phantom, (a) perpendicular to compression direction (parallel to detectors for the single angle PEM case), (b) normal to the detectors through the $9 \mathrm{~mm}$ sphere (normal to the AOR for PEM tomography) and (c) normal to the detectors through the 12 and $15 \mathrm{~mm}$ spheres (and normal to the AOR for PEM tomography). From left to right the columns are (1) PEM, $6.3 \mathrm{~cm}$ detector separation, (2) PEM, $8.0 \mathrm{~cm}$ detector separation, (3) PEM, $10.0 \mathrm{~cm}$ detector separation, (4) PEMT, $10 \mathrm{~cm}$ detector separation and $60^{\circ}$ rotation increment and (5) PEMT, $10 \mathrm{~cm}$ detector separation and $20^{\circ}$ rotation increment. The rows from top to bottom are with 4.0, 3.5, 3.0, 2.5 and $2.0 \mathrm{~mm}$ FWHM $3 \mathrm{D}$ post-reconstruction Gaussian filters. Each subimage slice is scaled to its own maximum.

angular acceptance windows with the $6.3 \mathrm{~cm}$ separation compared with the 8.0 and $10.0 \mathrm{~cm}$ separations.

\subsection{Analysis of angular sampling}

The maximum angular increment $\theta_{\max }$ for detector rotation that assures complete angular sampling of a point depends on its distance from the axis of rotation, the detector width and detector separation (equation (5)). This rotation increment decreases as the distance of a 
(a) SNR vs Contrast, $15 \mathrm{~mm}$ Sphere

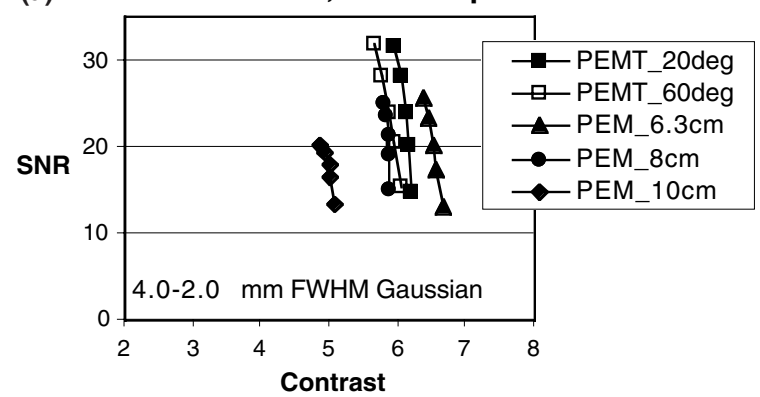

(b) SNR vs Contrast, $12 \mathrm{~mm}$ Sphere

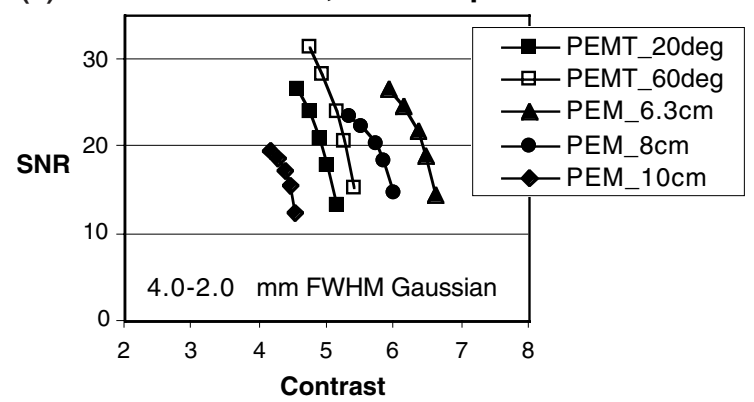

(c) SNR vs Contrast, $9 \mathrm{~mm}$ Sphere

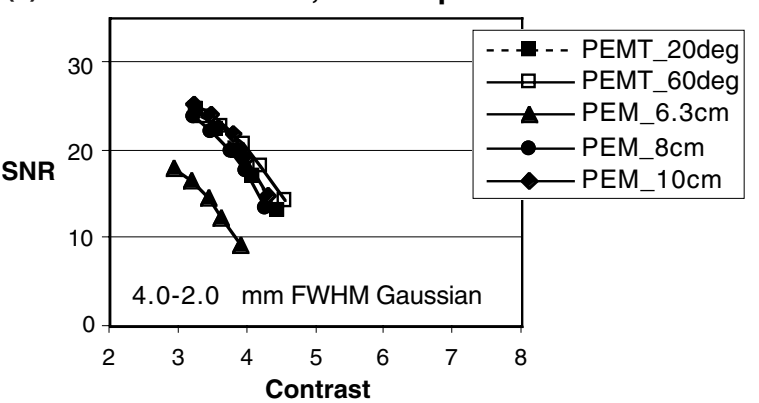

Figure 7. Signal-to-noise ratio (SNR) versus contrast for different post-reconstruction Gaussian smoothing filters for spherical tumour models in the compressed breast phantom. The angular rotation increment for the tomographic acquisitions and the front crystal separation for the static acquisitions are indicated in the legend. The leftmost data point of each curve is the result from a $4.0 \mathrm{~mm}$ FWHM filter, where SNR is greatest and contrast is least; the rightmost data point is the result from the $2.0 \mathrm{~mm}$ FWHM filter where SNR is least and contrast is greatest.

point from the axis of rotation increases and decreases as the distance between the detectors increases (figure 8). For the acquisition parameters of the breast phantom experiment, detector width $10 \mathrm{~cm}$ and detector separation $10 \mathrm{~cm}, \theta_{\max }$ for a point $4 \mathrm{~cm}$ from the AOR is $21.1^{\circ}$, consistent with observations (figure 3 ).

\section{Discussion}

This paper has demonstrated the application of PEM tomography using dual planar detectors; however, it also raises a number of intriguing questions, especially with respect to static 


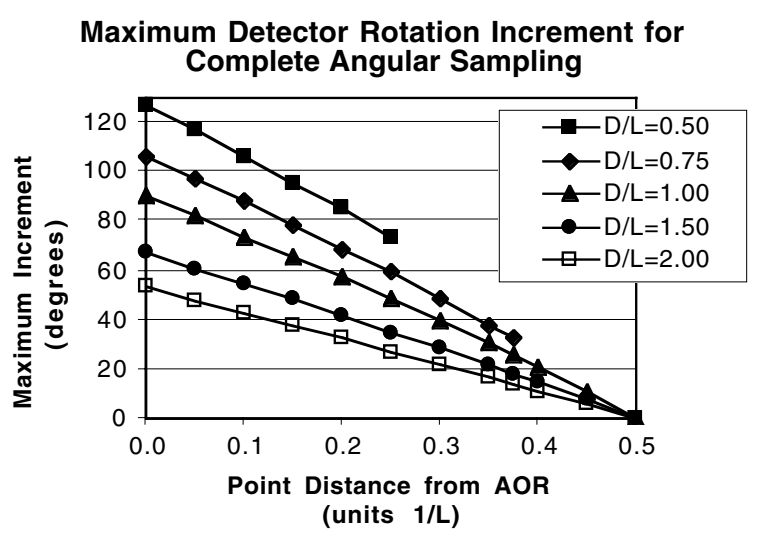

Figure 8. Maximum detector angular rotation increment $\theta_{\max }$ that maintains complete angular sampling of a point in the field of view (equation (5)) for $180^{\circ}$ detector rotation. The distance of the point from the axis of rotation is expressed in terms of the detector width $L$. The curves are for different values of the distance between the detectors, also expressed in units of $1 / L$.

PEM. For the compressed breast phantom experiment with 9, 12 and $15 \mathrm{~mm}$ diameter spheres and a 10:1 concentration ratio both PEM and PEMT perform quite well in terms of lesion visualization. Larger contrast values are achieved with PEM for the $12 \mathrm{~mm}$ and $15 \mathrm{~mm}$ spheres, while SNR is greatest for PEMT. It is not clear why contrast is greater with PEM, though perhaps it is due in part to a mismatch between the system matrix elements used in image reconstruction and the actual system matrix elements, which results in larger bias for limited angle tomography. The major unmodelled effects in the computation of the system matrix elements are scatter in the breast phantom, randoms, depth of interaction and the intrinsic detector resolution. The last-mentioned effect is due in part to Compton scatter in the crystal and electronics noise, which leads to crystal misidentification. This is evidenced in the non-zero valleys between adjacent crystals in the crystal identification map for our detector (Raylman et al 2000).

A comprehensive analysis of SNR and contrast for different sphere sizes, contrast ratios and detector separations has yet to be reported for any working PEM detectors for either PEM or PEMT operation. Image contrast results for 12 and $16 \mathrm{~mm}$ diameter hot-spot phantoms with hot-spot:background ratios of 4:1 to $55: 1$ for the $16 \mathrm{~mm}$ sphere and 33:1 to 80:1 for the $12 \mathrm{~mm}$ sphere have been reported for a PEM imager with a useful field of view of $5.9 \times$ $4.9 \mathrm{~cm}^{2}$, though image reconstruction was by the backprojection method and the measured hot-spot:background ratios were $1: 1$ to $14: 1$ for the $16 \mathrm{~mm}$ hot-spot and $4: 1$ to $14: 1$ for the $12 \mathrm{~mm}$ hot-spot (Murthy et al 1999). The detector separation for these measurements was not given, though it was probably close to the $7 \mathrm{~cm}$ thickness of the phantom. Our current paper represents an initial effort towards comparing PEM and PEMT, however further investigation is clearly warranted to determine how generalizable these results are. When comparing the performance of PEM and PEMT systems it is useful to keep in mind the results from simulation studies of Qi et al (2002), who remark that for large, high contrast lesions that are easy to identify, PEM with planar detectors and tomographic PEM with a rectangular detector may perform about the same if the area under the ROC curve is used as a performance measure.

Our current detector is rate-limited, so our results may change as faster acquisition systems are implemented. An increased data rate capability would provide an advantage to closer detector separations where the increased solid angle will improve sensitivity. We 
have tested a prototype VME-based data acquisition system that has less than $5 \%$ deadtime at a trigger rate of 10000 coincidences/s and about 25\% deadtime at 50000 coincidences/s, which is significantly better than the rates achievable with the current CAMAC-based system (Abbott et al 2002).

For both PEM and PEMT the choice of regularization filter affects contrast and SNR, with the range in contrast becoming greater as the size of the object decreases (figure 7). The choice of filter parameters will depend on the particular detection or quantitation task and will also be affected by the expected tumour-background ratio.

Depth of interaction effects degrade image quality, as illustrated here with point source reconstructions. A detector with depth of interaction capability has the potential to improve SNR (Qi et al 2002). Our current implementation of PEM and PEMT image reconstruction traces rays between points in the mid-crystal planes. The inclusion of depth of interaction effects in the system matrix element calculation should provide improved image quality. The mismatch between the true system matrix elements and those used in our current implementation due to depth of interaction effects will be greatest for raypaths with large incidence from normal and at smaller detector separations. These effects will also be most noticeable for smaller objects, which may account in part for the reduced SNR for $6.3 \mathrm{~cm}$ PEM (figure 7(c)). In the absence of DOI information from the PEM detector, it would be useful to study SNR versus contrast curves for different limitations on incidence angle. More isotropic PSFs are obtained by decreasing the allowable incidence angle from normal incidence, at the expense of sensitivity (e.g. figure 3). It is unclear what the relative trade-offs will be for different tumour sizes in clinical imaging conditions.

Future PEMT phantom studies could be performed with more anatomically realistic compressed breast phantoms of different sizes. The effect of body activity must be considered for clinical studies and we have made some initial studies of the effect of such activity for compressed breast phantoms (Raylman et al 2001b). A systematic contrast and SNR performance comparison of PEM and PEMT for compressed and pendant breast phantoms would be most interesting.

\section{Summary}

The feasibility of PEM tomography using dual planar detectors has been demonstrated in experiments with a compressed breast phantom and a compact GSO PEM system. For a fixed detector separation, initial evaluations show that PEMT generally provides improved image quality, SNR and contrast compared with PEM acquisition with stationary detectors. For this particular experiment with a high sphere:background activity concentration ratio, PEM with a smaller detector separation provides better contrast for 12 and $15 \mathrm{~mm}$ spheres while PEMT provides greater SNR. The SNR versus contrast curves are similar for all acquisition methods for the $9 \mathrm{~mm}$ diameter spheres. A formula was derived for the maximum detector rotation increment with step-and-shoot acquisition that maintains complete angular sampling. The detection or quantitation tasks for which PEMT or PEM is most appropriate remain a topic for more detailed future investigations.

\section{Acknowledgments}

The Southeastern Universities Research Association operates Thomas Jefferson National Accelerator Facility for the United States Department of Energy under contract DE-AC0584ER40150. This work is supported by the Office of Biological and Environmental Research 
of the Office of Science of the U.S. Department of Energy and by grant no R01 CA094196 from the National Cancer Institute of the National Institutes of Health. We thank Brian Kross, Vladimir Popov and Randolph Wojcik for their assistance with the detector electronics and mechanics.

\section{References}

Abbott D J, Weisenberger A, Majewski S, Kieper D, Kross B, Popov V and Wojcik R 2002 A high-performance VME-based acquisition system for positron emission mammography 2001 IEEE Nuclear Science Symposium Conference Record (San Diego, CA, 4-10 Nov. 2001) ed J A Seibert

Adler L P, Weinberg I N, Bradbury M S, Levine E A, Lesko N M, Geisinger K R, Berg W A and Freimanis R I 2003 Method for combined FDG-PET and radiographic imaging of primary breast cancers Breast J. 9 163-6

Doshi N K, Shao Y, Silverman R W and Cherry S R 2000 Design and evaluation of an LSO PET detector for breast cancer imaging Med. Phys. 27 1535-43

Freifelder R and Karp J S 1997 Dedicated PET scanners for breast imaging Phys. Med. Biol. 42 2463-80

Huesman R H, Klein G J, Moses W W, Qi J, Reutter B W and Virador P R G 2000 List-mode maximum-likelihood reconstruction applied to positron emission mammography (PEM) with irregular sampling IEEE Trans. Med. Imaging 19 532-37

Lange K and Carson R 1984 EM reconstruction algorithms for emission and transmission tomography J. Comput. Assist. Tomogr. 8 306-16

Levine E A et al 2003 Positron emission mammography: initial clinical results Ann. Surg. Oncol. 10 86-91

Levkovitz R, Falikman D, Zibulevsky M, Ben-Tal A and Nemirovski A 2001 The design and implementation of COSEM, an iterative algorithm for fully 3-D listmode data IEEE Trans. Med. Imaging $20633-42$

Moses W W and Qi J 2003 Fundamental limits of positron emission mammography Nucl. Methods Phys. Res. A 497 82-89

Muehllehner G, Buchin M P and Dudek J H 1976 Performance parameters of a positron imaging camera IEEE Trans. Nucl. Sci. 23 528-37

Murthy K, Aznar M, Thompson C J, Loutfi A, Lisbona R and Gagnon J H 2000 Results of preliminary clinical trials of the positron emission mammography system PEM-I: a dedicated breast imaging system producing glucose metabolic images using FDG $J$. Nucl. Med. 41 1851-8

Murthy K, Jolly D, Aznar M, Thompson C J, Sciascia P, Loutfi A, Lisbona R and Gagnon J H 1999 Quantification in positron emission mammography (PEM) with planar detectors: contrast resolution measurements using a custom breast phantom and novel spherical hot-spots IEEE Trans. Nucl. Sci. 46 2192-6

Nuyts J and Fessler J A 2003 A penalized-likelihood image reconstruction method for emission tomography, compared to postsmoothed maximum-likelihood with matched spatial resolution IEEE Trans. Med. Imaging 22 1042-52

Patton J A and Turkington T G 1999 Coincidence imaging with a dual-head scintillation camera J. Nucl. Med. 40 $432-41$

Qi J and Huesman R H 2002 Scatter correction for positron emission mammography Phys. Med. Biol. 47 2759-71

Qi J, Kuo C, Huesman R H, Klein G J, Moses W W and Reutter B W 2002 Comparison of rectangular and dual-planar positron emission mammography scanners IEEE Trans. Nucl. Sci. 49 2089-96

Raylman R R, Majewski S, Weisenberger A G, Popov V, Wojcik R, Kross B, Schreiman J S and Bishop H A 2001a Positron emission mammography-guided breast biopsy J. Nucl. Med. 42 960-66

Raylman R R, Majewski S, Wojcik R, Weisenberger A G, Kross B and Popov V 2001b Corrections for the effects of accidental coincidences, Compton scatter, and object size in positron emission mammography (PEM) imaging IEEE Trans. Nucl. Sci. 48 913-23

Raylman R R, Majewski S, Wojcik R, Weisenberger A G, Kross B, Popov V and Bishop H A 2000 The potential role of positron emission mammography for detection of breast cancer. A phantom study Med. Phys. 27 1943-54

Shepp L A and Vardi Y 1982 Maximum likelihood reconstruction for emission tomography IEEE Trans. Med.Imaging $1113-22$

Smith M F, Majewski S and Raylman R R 2002 Positron emission mammography with multiple angle acquisition 2002 IEEE Nuclear Science Symposium Conference Record (Norfolk, VA, 10-16 Nov. 2002) ed S Metzler

Smith M F, Majewski S, Weisenberger A G, Kieper D A, Raylman R R and Turkington T G 2003 Analysis of factors affecting positron emission mammography (PEM) image formation IEEE Trans. Nucl. Sci. 50 53-9

Thompson C J, Murthy K, Picard Y, Weinberg I N and Mako R 1995 Positron emission mammography (PEM): a promising technique for detecting breast cancer IEEE Trans. Nucl. Sci. 42 1012-7

Thompson C J, Murthy K, Weinberg I N and Mako F 1994 Feasibility study for positron emission mammography Med. Phys. 21 529-38 
Turkington T G, Majewski S, Weisenberger A G, Popov V, Smith M F, Sampson W H, Wojcik R and Kieper D 2002 A large field of view positron emission mammography imager 2002 IEEE Nuclear Science Symposium Conference Record (Norfolk, VA, 10-16 Nov. 2002) ed S Metzler

Virador P R G, Moses W W and Huesman R H 1998 Reconstruction in PET cameras with irregular sampling and depth of interaction capability IEEE Trans. Nucl. Sci. 45 1225-30

Weinberg I et al 1996 Preliminary results for positron emission mammography: real-time functional breast imaging in a conventional mammographic gantry Eur. J. Nucl. Med. 23 804-6

Weinberg I N, Stepanov P Y, Beylin D, Zavarzin V, Anashkin E, Lauckner K, Yarnall S, Doss M, Pani R and Adler L P 2002 PEM-2400 — a biopsy-ready PEM scanner with real-time x-ray correlation capability 2002 IEEE Nuclear Science Symposium Conference Record (Norfolk, VA, 10-16 Nov. 2002) ed S Metzler 